\title{
Management of Recurrent Acute Lymphoblastic Leukemia With T-Cell Engagement: CAR T, BiTEs, and Beyond
}

Presented by Jae Park, MD

\section{ABSTRACT}

Immunotherapies targeting CD19 (blinatumomab) and CD22 (inotuzumab ozogamicin) have demonstrated higher complete response rates and improved survival compared with chemotherapy in relapsed/refractory acute lymphoblastic leukemia (ALL), and are now standard of care in the relapsed setting. However, most adult patients still die of ALL despite these therapies, with or without hematopoietic stem cell transplant. At the NCCN 2019 Annual Congress: Hematologic Malignancies, Dr. Jae Park summarized clinical data from key trials of novel immunotherapies in ALL and reviewed evidence-based treatment approaches for adults with relapsed/refractory B-cell ALL.

J Natl Compr Canc Netw 2019;17(11.5):1448-1450

doi: $10.6004 /$ jnccn.2019.5030

T-cell engagement has led to key breakthroughs in the management of recurrent acute lymphoblastic leukemia (ALL). Despite these novel immunotherapies, however, up to $80 \%$ of adult patients still die of the disease with or without hematopoietic stem cell transplant (HSCT). At the NCCN 2019 Annual Congress: Hematologic Malignancies, Jae Park, MD, Associate Attending Physician, Memorial Sloan Kettering Cancer Center, summarized key studies of novel immunotherapies in relapsed/ refractory (R/R) ALL and discussed strategies that may improve the efficacy of these approaches. Dr. Park also reviewed evidence-based treatment approaches for adults with R/R B-cell ALL.

\section{Blinatumomab}

The effectiveness of blinatumomab, a bispecific T-cell engager antibody designed to direct cytotoxic $\mathrm{T}$ cells to CD19-expressing cancer cells, was demonstrated in the TOWER study, which compared blinatumomab to standard-of-care chemotherapy in R/R ALL, Dr. Park reported. ${ }^{1}$ Treatment with blinatumomab resulted in significantly longer overall survival compared with chemotherapy among adult patients with R/R B-cell precursor ALL. Subgroup analysis also showed a trend toward improved survival in patients with higher versus lower tumor burden. Nevertheless, although patient outcomes were improved, overall survival for those randomized to blinatumomab was still only 7.7 months (vs 4.0 months for chemotherapy).

"We need to do better for these patients," said Dr. Park, who noted that blinatumomab is currently being studied in the frontline setting in older patients with newly diagnosed B-ALL. Preliminary data presented at the 2018 American Society of Hematology Annual Meeting showed a complete response rate of approximately $66 \%$ (Figure 1). ${ }^{2}$ These results are "very encouraging for single-agent blinatumomab," said Dr. Park, but longer follow-up is needed. Moreover, comparatively few study patients have made it to transplantation due to their age and fitness level.

As Dr. Park reported, minimal residual disease (MRD) status has become an important prognostic indicator in the treatment of ALL. In a study of adults with MRD-positive ALL in hematologic remission after chemotherapy, $78 \%$ achieved a complete MRD response with blinatumomab, allowing most of the responding patients to proceed to transplantation in better remission status. ${ }^{3}$ Based on these data, blinatumomab was FDA-approved for use in MRD-positive B-cell ALL in either first or second remission with at least $0.1 \%$ MRD.

\section{Inotuzumab Ozogamicin}

Another treatment approach for R/R B-cell ALL is inotuzumab ozogamicin (IO), an anti-CD22 antibody conjugated to calicheamicin. As Dr. Park explained, this drug provides very selective delivery of chemotherapy. Results of the INO-VATE trial demonstrated higher response rates for IO versus chemotherapy ( $\sim 80 \%$ vs $\sim 30 \%$ ), and a higher percentage of patients randomized to the IO group experienced MRD-negative remission (78\% vs 28\%). "More than $40 \%$ of patients were able to progress to HSCT compared with just $11 \%$ of those who received 


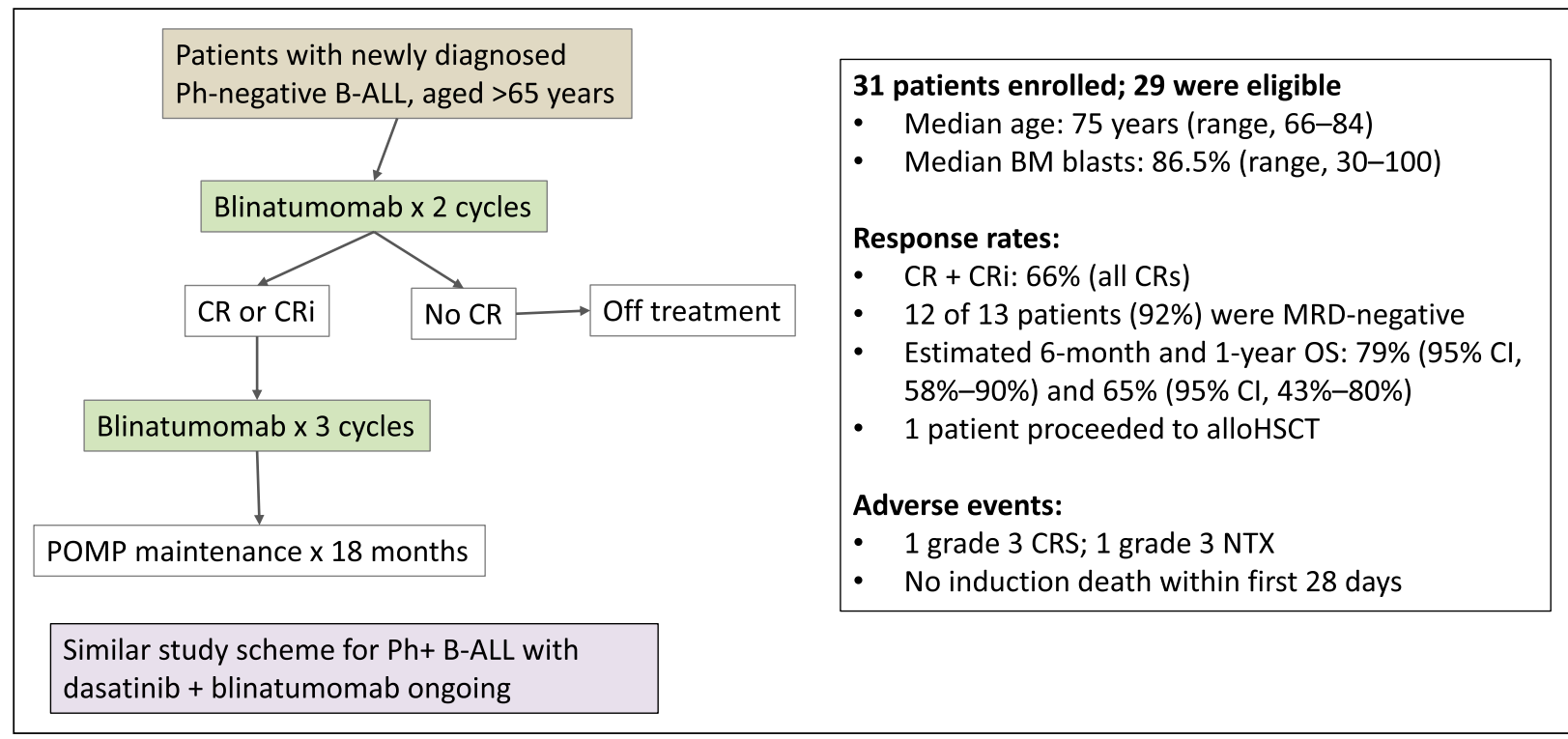

Figure 1. Study schema for SWOG 1318 examining blinatumomab in the frontline setting for older adults.

Abbreviations: alloHSCT, allogeneic hematopoietic stem cell transplantation; B-ALL, B-cell acute lymphoblastic leukemia; BM, bone marrow; CR, complete response; $\mathrm{CRi}$, incomplete hematologic response; CRS, cytokine release syndrome; MRD, minimal residual disease; OS, overall survival; Ph, Philadelphia chromosome; POMP, prednisone/vincristine/methotrexate/6-mercaptopurine.

Data from Advani AS, Moseley A, O'Dwyer KM, et al. Results of SWOG 1318: a phase 2 trial of blinatumomab followed by POMP (prednisone, vincristine, methotrexate, 6-mercaptopurine) maintenance in elderly patients with newly diagnosed Philadelphia chromosome negative B-cell acute lymphoblastic leukemia [abstract]. Blood 2018;132(Suppl 1):Abstract 33.

standard chemotherapy," said Dr. Park. He also noted, that veno-occlusive disease (VOD) was a major adverse event associated with IO.

In patients for whom HSCT is considered, said Dr. Park, the number of IO cycles should be limited to 2 , if feasible, and conditioning regimens with dual alkylating agents (eg, thiotepa, melphalan) should be avoided. In addition, ursodiol should be given to all patients exposed to IO, and bilirubin level, liver function, and weight should be measured and noted before each IO dose to monitor for VOD. IO is approved for treatment of patients with relapsed B-cell ALL, and is being studied in young adults with newly diagnosed CD22-positive, Phnegative B-cell ALL.

\section{CAR T Cells}

CAR (chimeric antigen receptor) $\mathrm{T}$ cells provide another treatment strategy against R/R B-cell ALL. Using this approach, $\mathrm{T}$ cells are isolated from the individual patient and genetically engineered to express a CAR that targets a specific tumor antigen, such as CD19. These CAR-modified T cells are then activated and expanded in culture before being infused back into the patient.

In a global study of CD19 CAR T-cell therapy using tisagenlecleucel, a single infusion of CD19 CAR therapy provided durable remission with long-term persistence in pediatric and young adult patients (aged $\leq 25$ years) with R/R B-cell ALL. ${ }^{5}$ The overall response rate was $81 \%$, with 8 patients $(11 \%)$ proceeding to post-CAR allogeneic HSCT. Tisagenlecleucel was subsequently approved by the FDA for this age group.

In a similar study with a different CD19 CAR T-cell construct, ${ }^{6}$ Dr. Park and colleagues at Memorial Sloan Kettering Cancer Center demonstrated comparable findings in adults with R/R B-cell ALL, but there are still no approved products for use in adult patients.

\section{Unique Toxicities of T Cell-Activating Immunotherapy}

Although immunotherapeutic agents have resulted in significantly improved response rates and survival in the relapsed setting compared with chemotherapy, serious toxicities are associated with immune activation. The main toxicity, explained Dr. Park, is cytokine release syndrome (CRS), which manifests similarly to sepsis and is characterized by fever, hypotension, capillary leak, respiratory insufficiency, and, in severe cases, hemophagocytic lymphohistiocytosis. Immune effector cellassociated neurotoxicity syndrome (ICANS) is another concern, which typically manifests with delirium and aphasia and can progress to global encephalopathy and seizures, and in severe cases, rapid onset cerebral edema has been observed.

With blinatumomab, said Dr. Park, these toxicities can occur within the first few days of treatment. Data from the TOWER study showed that $9 \%$ and $5 \%$ of patients 


\begin{tabular}{|l|l|c|c|c|c|c|}
\hline \multicolumn{1}{|l|}{} & T-Cell Product & Disease & $\begin{array}{c}\text { Patients } \\
\text { N }\end{array}$ & $\begin{array}{c}\text { CRS, } \\
\text { All Grades }\end{array}$ & $\begin{array}{c}\text { Grade } \geq 3 \\
\text { CRS }\end{array}$ & $\begin{array}{c}\text { Grade } \geq 3 \\
\text { NTX }\end{array}$ \\
\hline \multirow{5}{*}{ Adults } & $\mathbf{1 9 - 2 8 z ~ ( M S K ) ~}$ & ALL & 53 & $85 \%$ & $26 \%$ & $41 \%$ \\
\cline { 2 - 7 } & KTE-C19 (ZUMA-3) & ALL & 29 & $93 \%$ & $28 \%$ & $52 \%$ \\
\cline { 2 - 7 } & KTE-C19 (ZUMA-1) & DLBCL & 101 & $93 \%$ & $13 \%$ & $28 \%$ \\
\cline { 2 - 8 } & CTL019 (JULIET) & DLBCL & 99 & $58 \%$ & $23 \%$ & $12 \%$ \\
\cline { 2 - 8 } & JCAR017 (TRANSCEND) & DLBCL & 55 & $35 \%$ & $2 \%$ & $16 \%$ \\
\hline \multirow{3}{*}{ Peds } & CTL019 (ELIANA) & ALL & 75 & $77 \%$ & $47 \%$ & $13 \%$ \\
\cline { 2 - 8 } & JCAR017 (PLAT-02) & ALL & 43 & $93 \%$ & $23 \%$ & $21 \%$ \\
\cline { 2 - 7 } & $\mathbf{1 9 - 2 8 z ~ ( N C I ) ~}$ & ALL & 21 & $76 \%$ & $29 \%$ & $5 \%$ \\
\hline
\end{tabular}

Figure 2. CD19 CAR-associated CRS and NTX incidence in ALL.

Abbreviations: ALL, acute lymphoblastic leukemia; CRS, cytokine release syndrome; DLBCL, diffuse large B-cell lymphoma.

experienced neurologic events and CRS, respectively, most of which were low-grade and resolved spontaneously or with dose interruptions. ${ }^{1}$ With CAR T-cell therapy, CRS and ICANS typically occur within the first 1 to 2 weeks of infusion, but the incidence and severity are much higher. Safety data from multiple CAR T-cell trials suggest that, on average, $30 \%$ to $40 \%$ of patients experience grade $\geq 3$ CRS and neurotoxicity, but incidence and severity vary depending on the patients enrolled and disease burden at the time of T-cell infusion. (Figure 2).

Despite the severity and frequency of these symptoms, however, Dr. Park emphasized that both
CRS and neurologic toxicities are reversible and can be managed with tocilizumab (IL-6R inhibitor) and/or corticosteroids.
Disclosures: Dr. Park has disclosed that he receives grant/research support from Amgen Inc., Genentech, Inc., Incyte Corporation, Juno Therapeutics, Inc. Kite Pharma, Novartis Pharmaceuticals Corporation, and Servier; and is a scientific advisor for from Amgen Inc., AstraZeneca Pharmaceuticals LP,

GlaxoSmithKline, Incyte Corporation, Kite Pharma, Novartis Pharmaceuticals Corporation, Allogene Therapeutics, Autolus Therapeutics plc, and Takeda Pharmaceuticals North America, Inc.

Correspondence: Jae Park, MD, Memorial Sloan Kettering Cancer Center 1275 York Avenue, New York, NY 10065. Email: parkj6@mskcc.org

\section{References}

1. Kantarjian $\mathrm{H}$, Stein A, Gökbuget N, et al. Blinatumomab versus chemotherapy for advanced acute lymphoblastic leukemia. N Engl J Med 2017;376:836-847.

2. Advani AS, Moseley A, O'Dwyer KM, et al. Results of SWOG 1318: a phase 2 trial of blinatumomab followed by POMP (prednisone, vincristine, methotrexate, 6-mercaptopurine) maintenance in elderly patients with newly diagnosed Philadelphia chromosome negative B-cell acute lymphoblastic leukemia [abstract]. Blood 2018;132(Suppl 1):Abstract 33.

3. Gökbuget $N$, Dombret $\mathrm{H}$, Bonifacio $\mathrm{M}$, et al. Blinatumomab for minimal residual disease in adults with B-cell precursor acute lymphoblastic leukemia. Blood 2018;131:1522-1531.

4. Kantarjian HM, DeAngelo DJ, Stelljes M, et al. Inotuzumab ozogamicin versus standard therapy for acute lymphoblastic leukemia. N Engl J Med 2016;375:740-753

5. Maude SL, Laetsch TW, Buechner J, et al. Tisagenlecleucel in children and young adults with B-cell lymphoblastic leukemia. N Engl J Med 2018 378:439-448.

6. Park JH, Riviere I, Gonen M, et al. Long-term follow-up of CD19 CAR therapy in acute lymphoblastic leukemia. N Engl J Med 2018;378: 449-459 